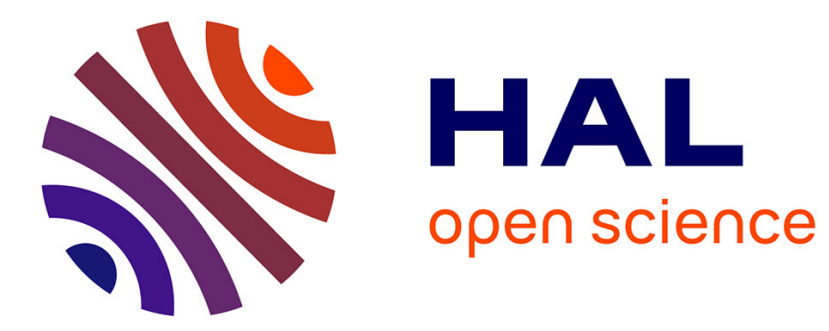

\title{
Éléments statistiques sur cinquante ans d'aide européenne au développement
}

François Pacquement

\section{To cite this version:}

François Pacquement. Éléments statistiques sur cinquante ans d'aide européenne au développement.

La France, l'Europe et l'aide au développement - des traités de Rome à nos jours, 2013. hal-01984635

\section{HAL Id: hal-01984635 \\ https://hal.science/hal-01984635}

Submitted on 17 Jan 2019

HAL is a multi-disciplinary open access archive for the deposit and dissemination of scientific research documents, whether they are published or not. The documents may come from teaching and research institutions in France or abroad, or from public or private research centers.
L'archive ouverte pluridisciplinaire HAL, est destinée au dépôt et à la diffusion de documents scientifiques de niveau recherche, publiés ou non, émanant des établissements d'enseignement et de recherche français ou étrangers, des laboratoires publics ou privés. 


\section{Éléments statistiques sur cinquante ans d'aide européenne au développement}

Dès 2005, les Etats membres de l'union européenne se sont donné pour objectif de consacrer à l'aide au développement $0,7 \%$ de leur $\mathrm{RNB}$, engagement singulièrement fort dans la communauté du développement. L'analyse quantitative de l'aide européenne, à travers 50 ans de statistiques, permet d'apprécier la contribution des Etats-membres à travers leurs institutions communes.

\section{L'aide publique au développement est mesurée par le Comité d'aide au développement de l'OCDE.}

Définie dans les années 1960, l'aide publique au développement (APD) est mesurée par l'Organisation de coopération et de développement économiques (OCDE). Créée en 1961, l'OCDE est l'héritière de l'OECE (Organisation européenne de coopération économique), elle-même instituée après la seconde guerre mondiale pour réunir les Etats-Unis et les pays bénéficiaires du Plan Marshall. Ainsi, au départ, certains membres de l'OCDE ont eux-mêmes été des bénéficiaires d'aide. Aujourd'hui, l'Organisation constitue un club, au sein duquel les pays industriels débattent des divers aspects de leur gestion publique afin d'y définir de " bonnes pratiques ". L'Organisation ne consent pas de financement, l'emploi du terme " développement" visant à souligner une préoccupation alors naissante de la coopération internationale.

La Direction de la coopération au développement assure le secrétariat du Comité d'aide au développement (CAD), qui vise à promouvoir l'expansion de l'aide consentie par les pays membres en direction des pays en développement et à améliorer l'efficacité des ressources allouées. Si les membres fondateurs n'étaient qu'au nombre de neuf (Allemagne, Belgique, Canada, Etats-Unis, France, Italie, Portugal ${ }^{1}$, Royaume-Uni et la Commission de la Communauté économique européenne), le CAD compte aujourd'hui vingt-quatre membres (Australie, Canada, Corée, Etats-Unis, Japon, Norvège, Nouvelle-Zélande, Suisse, ainsi que les grands pays donateurs de l'Union Européenne ${ }^{2}$, auxquels s'ajoute la représentation de la Commission Européenne).

Le CAD constitue un élément important d'encadrement administratif et politique de l'Aide publique au développement. Il procède à un examen régulier entre pairs des systèmes de coopération et de la politique d'aide au développement des pays membres. Il comptabilise les efforts d'aide, formule des recommandations afin d'accroître leur efficacité ("bonnes pratiques" de l'aide, agenda pour la coopération au XXIème siècle, etc.) et en vérifie l'application à l'occasion des examens entre pairs.

La définition de l'aide publique au développement (APD) est arrêtée en 1969 ; elle désigne toute dépense publique assortie des trois caractéristiques suivantes :

- s'adresser à un pays en développement (PED) éligible, selon une liste préétablie ;

- favoriser le développement économique et l'amélioration des conditions de vie dans le pays concerné ;

- comporter un élément de libéralité minimum.

L'élément de libéralité d'un prêt désigne l'écart entre le montant du prêt (le principal) et les remboursements prévisionnels actualisés (écart exprimé en pourcentage du principal). Un élément de libéralité de $40 \%$ indique donc que le remboursement du prêt équivaut à une valeur actuelle de $60 \%$ du principal. Plus la durée est longue, plus l'échéance du premier remboursement est éloignée, plus le taux d'intérêt est bas, plus la libéralité augmente. Lors du remboursement, l'aide est diminuée à hauteur de l'échéance en principal effectivement acquittée.

1 En 1974, le Portugal s'est retiré du CAD et a demandé à être inscrit sur la liste des pays en développement établie par le CAD ; le Portugal a réintégré le CAD en 1991.

2 Soit 15 pays: Allemagne, Autriche, Belgique, Danemark, Espagne, Finlande, France, Grèce, Irlande, Italie, Luxembourg, Pays-Bas, Portugal, Royaume-Uni et Suède. 
Sont également comptabilisées comme APD l'aide alimentaire et l'aide d'urgence, l'aide aux réfugiés, les remises de dettes, certaines opérations précises de maintien de la paix (remise en état d'infrastructures, acheminement des secours d'urgence, déminage, démobilisation, organisation d'élections, etc.).

L'aide européenne comporte toutes ces formes d'intervention, et se fait sous forme de subventions distribuées par la Commission et de prêts de la Banque européenne d'investissement.

\section{L'aide au développement se répartit entre le bilatéral et le multilatéral ; l'aide européenne est classée dans l'effort multilatéral}

L'aide peut être consentie soit directement par un Etat (aide bilatérale), soit par l'intermédiaire d'un organisme international (aide multilatérale). La répartition entre ces deux modes d'acheminement s'est stabilisée depuis 1975 ; depuis cette année, l'aide multilatérale constitue autour de $30 \%$ de l'APD globale de tous les pays de l'OCDE.

Part de l'aide bilatérale et de l'aide multilatérale dans l'aide totale

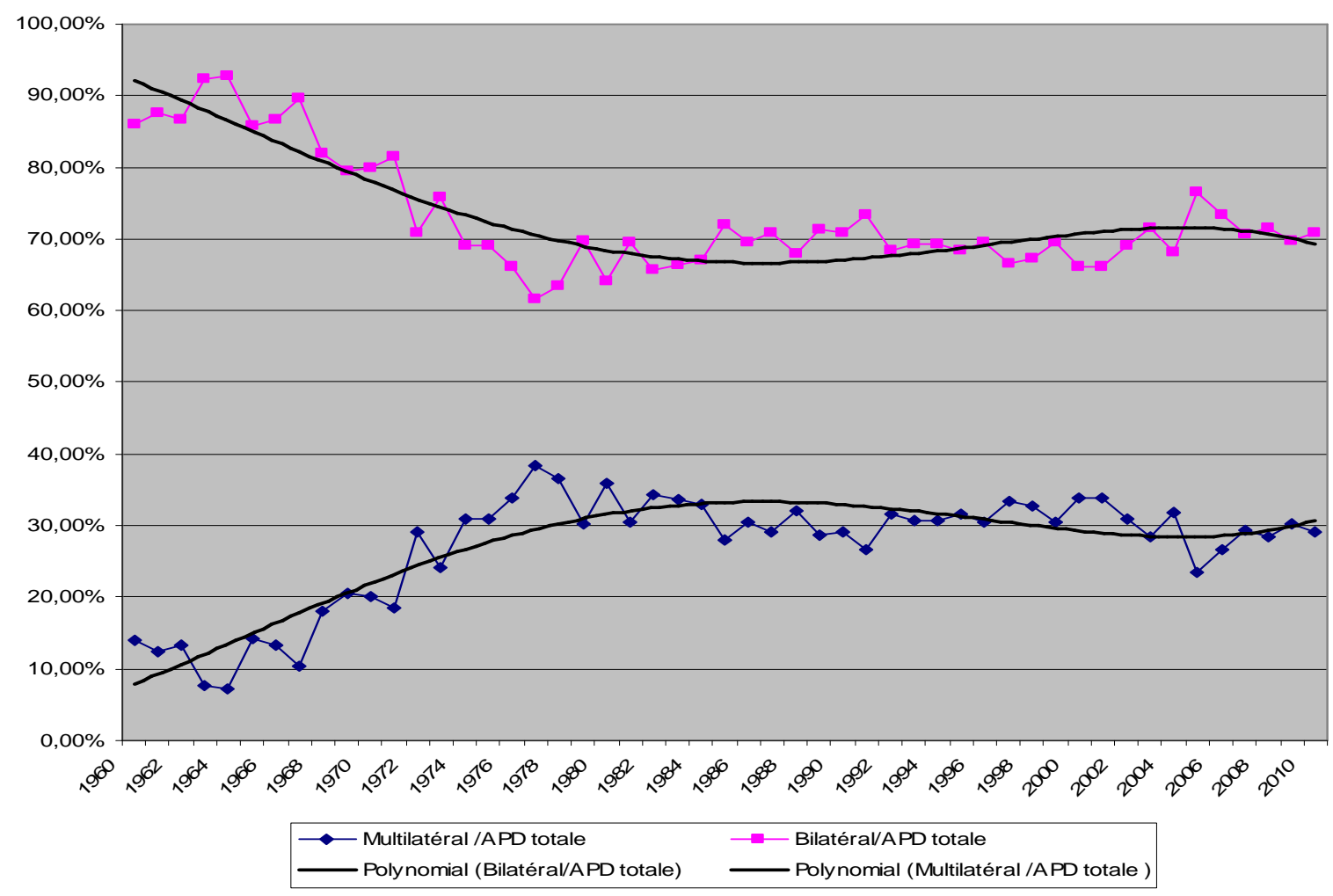


Au sein de l'aide multilatérale, l'aide européenne voit sa part augmenter continûment.

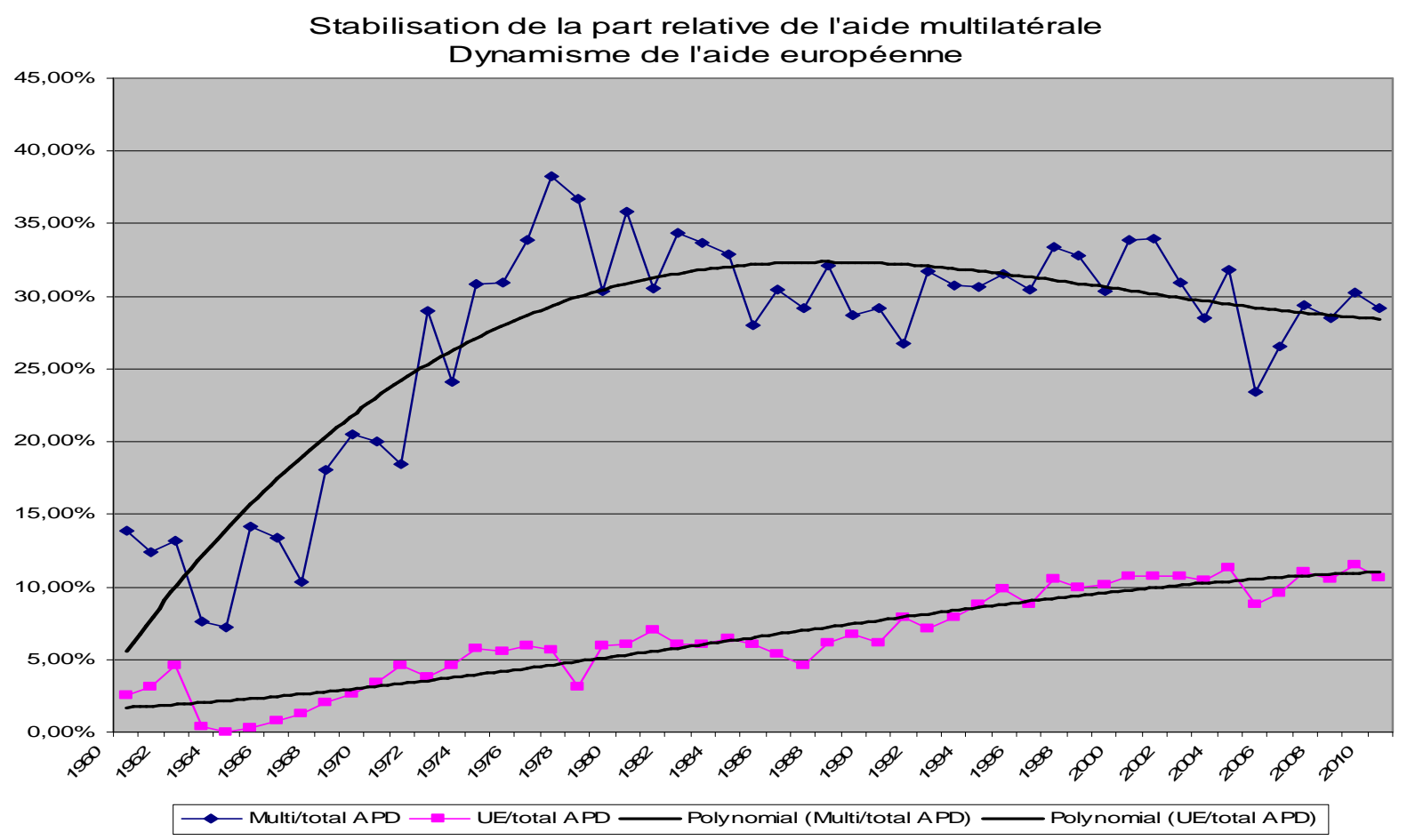

\section{L'évolution en montant de l'aide européenne fait apparaître quelques caractéristiques}

Une croissance soutenue caractérise l'aide européenne, qui résiste même à la crise de l'aide des années 1990- pendant cette décennie, ce sera la seule aide à connaittre une croissance soutenue.

L'aide des institutions européennes se compare à celle des Etats-membres (millions de dollars 2009)

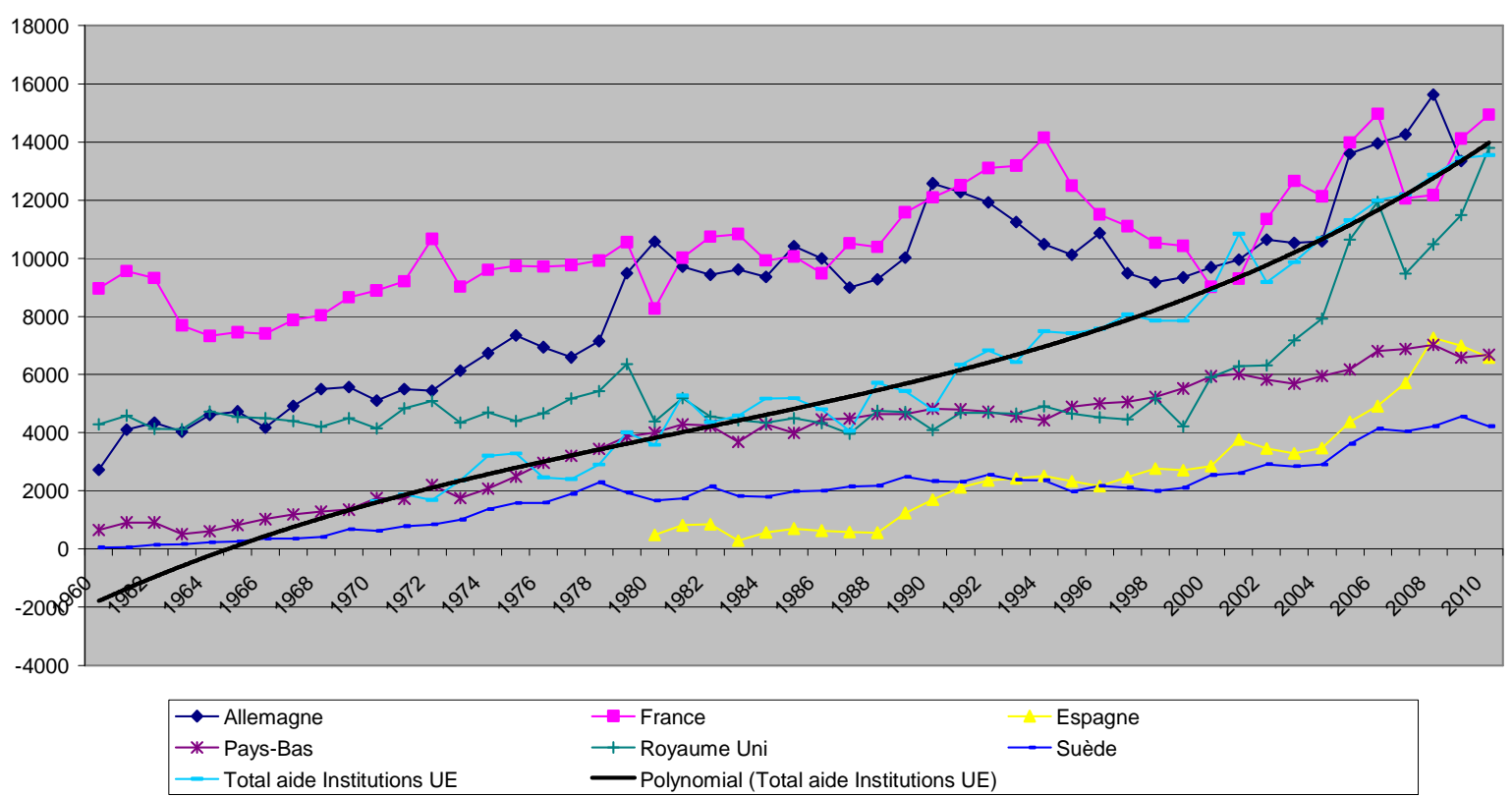




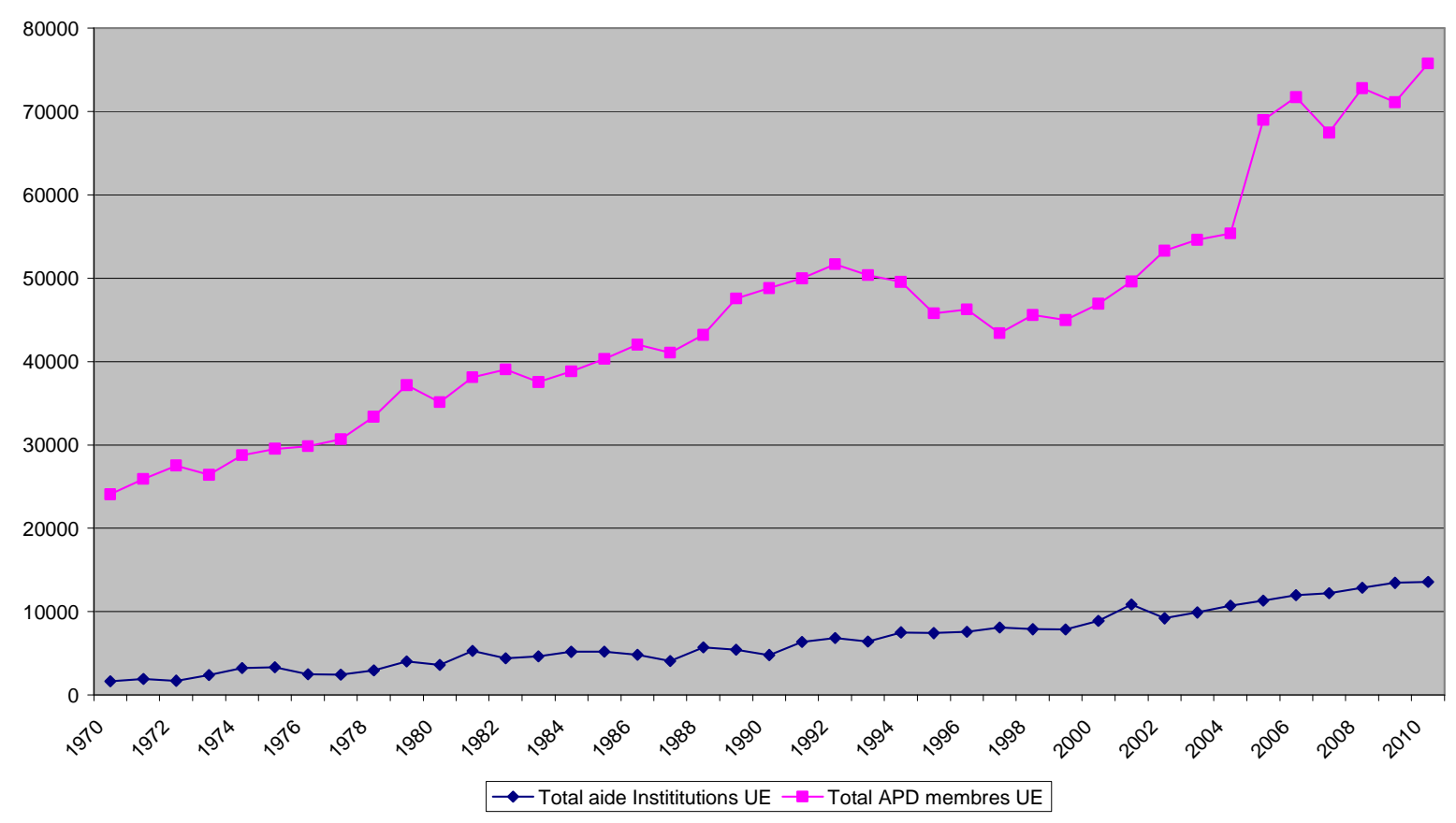

La répartition géographique fait apparaitre une érosion lente de la part de l'Afrique. L'aide à l'Europe diminue avec l'élargissement aux pays d'Europe centrale et orientale, au profit de l'Asie.

\section{Répartition géographique de l'aide par continent}

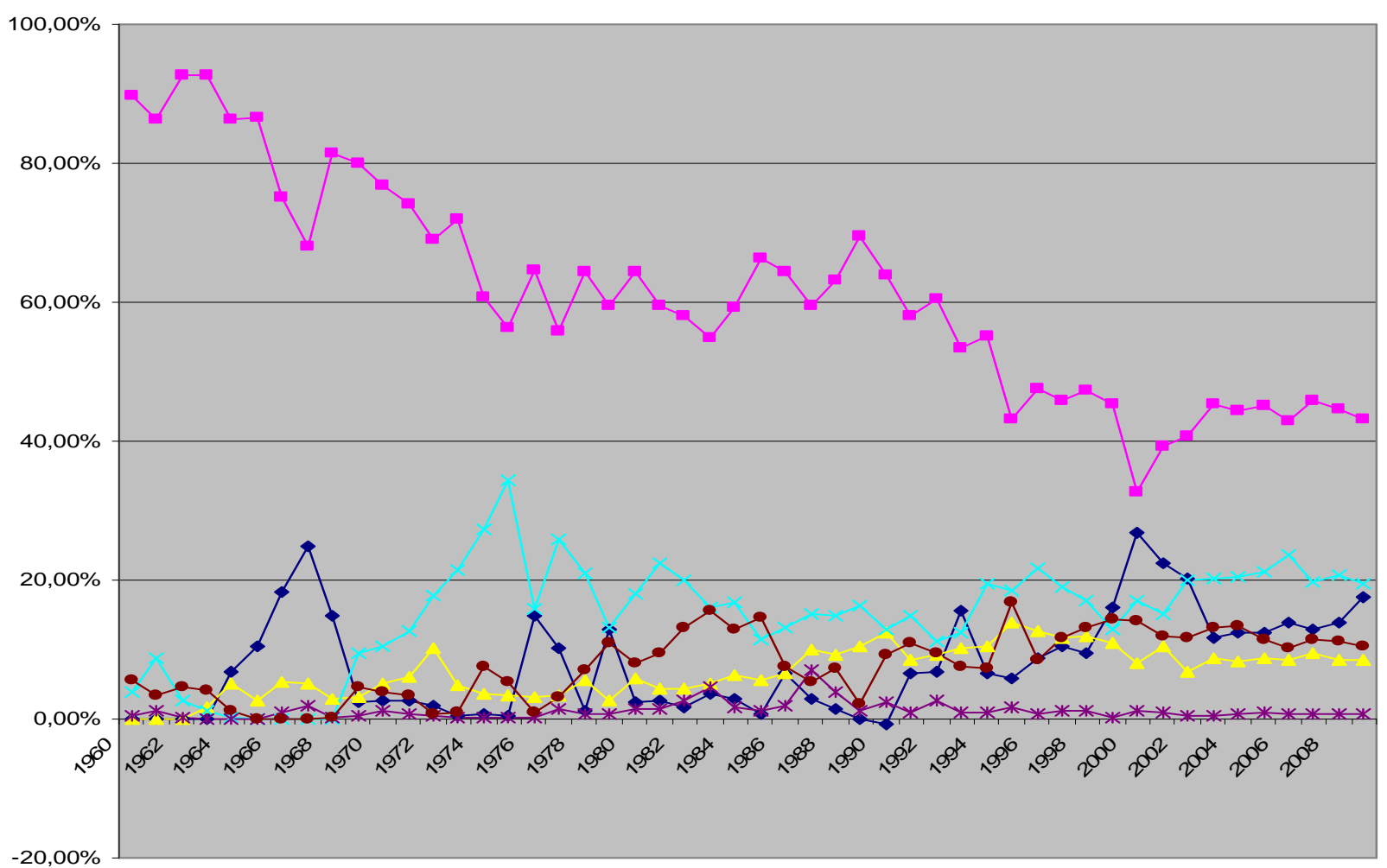


La part des pays les moins avancés (PMA) et autres pays à faible revenu (PFR) baisse continûment jusqu'en 2001, puis elle ré augmente après l'adoption des « Objectifs de développement du millénaire ».

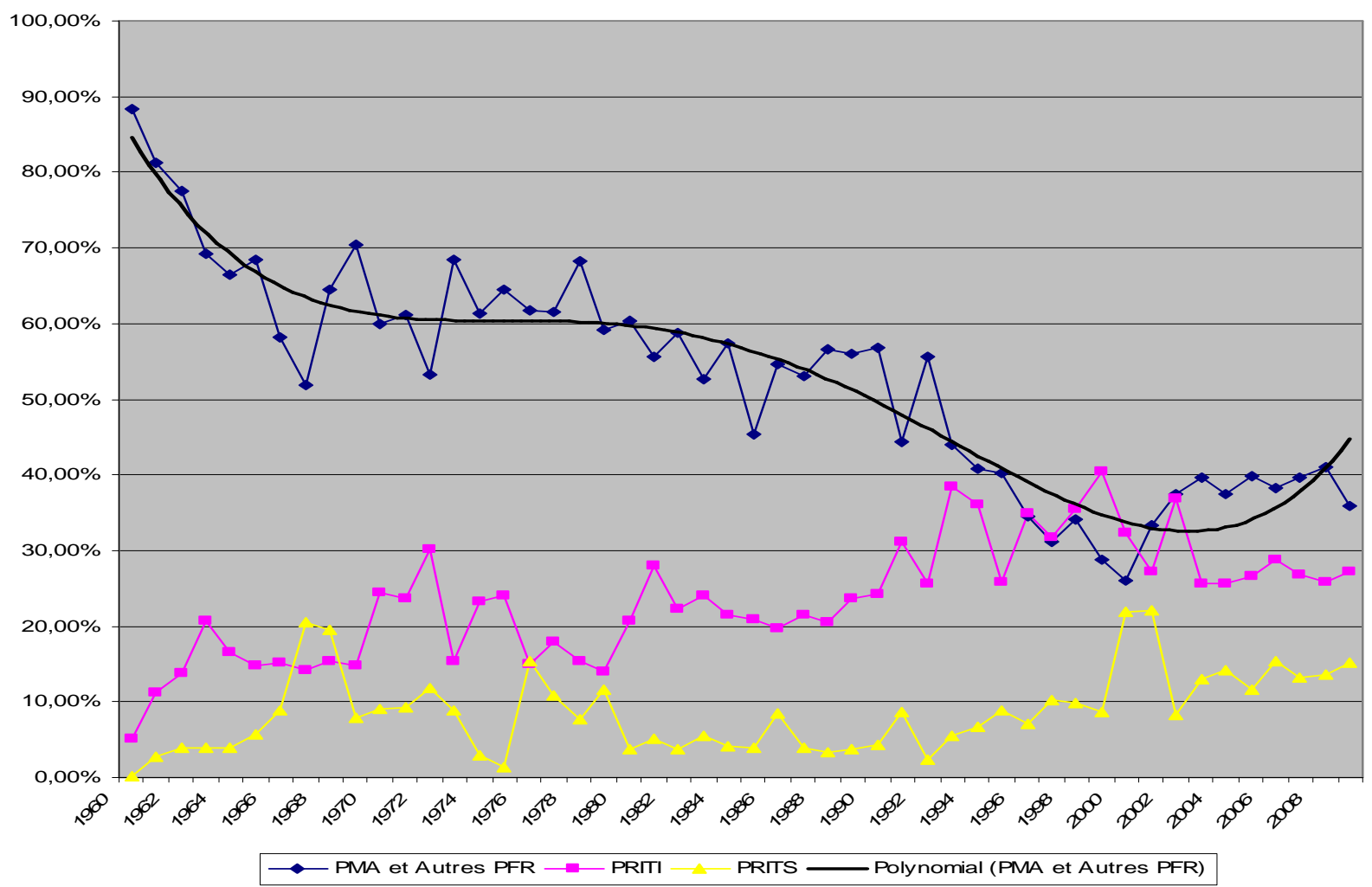

Au total, l'aide européenne au développement connait des évolutions en montants qui semblent continues, malgré les diverses évolutions en termes soit de nombre d'Etats-membres, soit d'événement historique. Cette aide, remarquablement stable, résiste à la concurrence des autres aides multilatérales. Elle progresse continûment à travers ses trois grands moments, les débuts (1957-1975), les élargissements et nouveaux paradigmes de l'aide (1975-1995) et la remise en cause de l'approche européenne du développement (1995-2010). C'est sans doute là une qualité remarquable de l'aide européenne.

\section{Bibliographie}

COMMISSION STAFF WORKING DOCUMENT Brussels, 21.4.2010 SEC(2010) 420 final Financing for Development - Annual progress report 2010 Getting back on track to reach the EU 2015 target on ODA spending?

Organisation de Coopération et de Développement Économiques DCD/DAC(2011)21/FINAL 08-Nov20112011 DAC REPORT ON MULTILATERAL AID

François Pacquement Bâtir des politiques globales: l'aide au développement, source d'inspiration? Afrique contemporaine 2009/3 ( $\mathrm{n}^{\circ}$ 231) (version anglaise : Building global policies: development assistance, a source of inspiration? - IDGM [Sciences Po- Université d'Auvergne] - octobre 2010)

Julie Walz and Vijaya Ramachandran Brave New World A Literature Review of Emerging Donors and the Changing Nature of Foreign Assistance 